\title{
Online intelligent controlled mine detecting robot
}

\author{
K.Prema \\ Department of ICE \\ SRM university,Chennai, \\ India
}

\author{
N.Senthil kumar \\ Department of EEE \\ Mepco schlenk engineering \\ college \\ Sivakasi, India
}

\author{
S.S. Dash \\ Department of EEE \\ SRM university,Chennai, \\ India
}

\author{
S.Siva Chandran \\ Department of ICE \\ SRM university, Chennai, \\ India
}

\begin{abstract}
This paper proposes the design of a micro-controller based fuzzy logic controller for a remote controlled mine detecting robot. In the real time applications the detection and location of bomb is highly essential in the field of defense applications. Considering the value of human life the robot is allowed in the field to detect the bomb. The mine detecting robot is designed with IR sensors, metal detector and GPS attached to it.

The two DC motors are connected with the rear wheels of the robot. Differential drive is used to control the steering angle and the speed of the robot. Differential drive is a method of controlling a robot with only two motorized wheels. They are controlled by a fuzzy logic controller to offer accurate steering angle and the driving speed of the robot. The designed controller has two loops with an Outer Fuzzy Speed Control Loop and an Inner Current Control Loop. Based on the current position and the set speed value, the steering angle and the speed of a mine detecting robot will be controlled. The software for both the client system and the robot is developed using Data socket protocol in LabVIEW. The motion of the robot is monitored by RF camera. The designed controller was implemented in a PIC 16F877A microcontroller and the results are documented. The mine is detected by metal detector and the area of the mine is known through GPS.
\end{abstract}

\section{General Terms}

Algorithms performance.

\section{Keywords}

LabVIEW; FuzzyController; Data Socket Protocol; GPS.

\section{INTRODUCTION}

LabVIEW is one of the well-known software packages used in process control applications. Dc motor are used in control applications especially robotics motion control. The speed of the Dc motor is normally controlled by varying armature voltage and flux. In armature controlled dc motor the desired speed is obtained by varying armature voltage. However, conventional proportional integral derivative (PID) control has difficulty in dealing with dynamic speed tracking due to parameter variations, and load disturbances. Hence these controllers show high performance only for one unique act point. The fuzzy logic controller (FLC) provides a systematic way to incorporate the human intelligence in the controller without knowing the mathematical model of the system. The stability of the system and wide range of operating speed are achieved through fuzzy logic controller. When the optimum membership functions are chosen for input and output of the FLC then it works with self-tuning capability and its stability depends upon rule base.

\section{PROPOSED SYSTEM:}

The goal of the proposed system is to prepare an outdoor mobile robot platform for a humanitarian demining application. The system consists of pic microcontroller with inbuilt $\mathrm{A} / \mathrm{D}$ converter which is configured as bomb detecting robot. The global positioning system (GPS) is attached to robot to trace the position of the robot. The client system with network interface card (NIC) is the user of the robot. The client and the robot are communicated through internet.

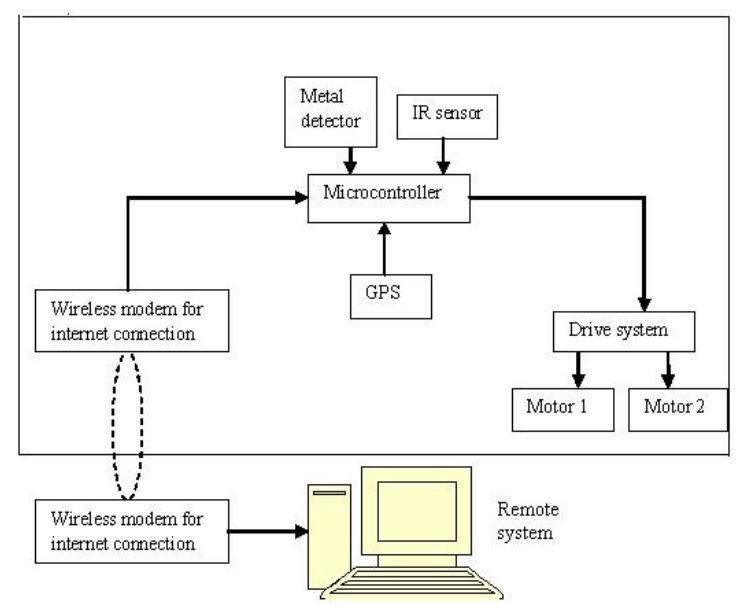

Fig 1: Internet based control setup

In client system, the data socket protocol in LAB VIEW serves as the communication tool for internet. The bomb detecting Robot is connected to the server computer using a DAQ board and its IP address is noted. The specified direction of the robot by means of LAB VIEW is transmitted from client system to the robot through internet. The $\mathrm{h}$ bridge converter is used to supply voltage to DC motor according to the direction. Differential drive is used to control the steering angle and the speed of the robot. Differential drive is a method of controlling a robot with only two motorized wheels. The two DC motors are connected with the rear wheels of the robot. The speed of the motor is controlled automatically to the user specified speed by the fuzzy logic controller. The designed controller has two loops with an Outer Fuzzy Speed Control Loop and an Inner Current Control Loop. The IR sensor and the metal detector in the robot serve as the obstacle sensor and bomb detection respectively. The IR sensor status and metal detector sensor status are transmitted to the client system from robot through internet. The status of IR sensor helps for path planning and changing the direction of robot. When the bomb is detected by metal detector the robot is stopped and the location of bomb is identified with the help of GPS. Figure1. Shows the Internet based control setup of the proposed system. 


\section{MATHEMATICAL MODEL OF DC MOTOR:}

To be modeling a DC Motor, simple circuit of its electrical diagram as shown in Figure 2. is considered. [1-2], to be Modeling and Simulate the DC motor, the following steps are to be made step by step; [10][16]

Step1: Represent the DC motor circuit diagram.

Step2: Represent system equations.

Step3: obtain the state space model.
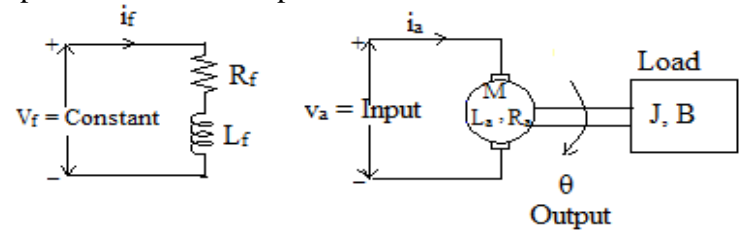

Fig2: DC motor

\subsection{Loop System Consideration:}

To perform the simulation of the system, an appropriate model needs to be established. Therefore, a model based on the motor specifications needs to be obtained. Figure2 Shows the DC motor circuit with Torque and Rotor Angle Consideration.

\subsection{System equation:}

The motor torque $\mathbf{T}$ is related to the armature current, $\mathbf{i}$, by a torque constant $\mathbf{K}$;

$\mathbf{T}=\mathbf{K}_{\mathbf{i}}$

(1)

The generated voltage, ea, is relative to angular velocity by;

$$
e_{a}=K \omega_{m}=K \frac{d \theta}{d t}
$$

From Figure 2 we can write the following equations based on the Newton's law combined with the Kirchhoff's law:

$$
\begin{gathered}
J \frac{d^{2} \theta}{d t^{2}}+b \frac{d \theta}{d t}=K i \\
L \frac{d i}{d t}+R i=V-K \frac{d \theta}{d t}
\end{gathered}
$$

\subsection{State-Space model for DC Motor:}

The dynamic equations in state-space form are the following:

$$
\begin{gathered}
\frac{\mathrm{d}}{\mathrm{dt}}\left[\begin{array}{c}
\theta \\
\dot{\theta} \\
\mathrm{i}
\end{array}\right]=\left[\begin{array}{rrr}
0 & 1 & 0 \\
0 & -\frac{\mathrm{b}}{\mathrm{J}} & \frac{\mathrm{K}}{\mathrm{T}} \\
0 & -\frac{\mathrm{k}}{\mathrm{L}} & -\frac{\mathrm{h}}{\mathrm{L}}
\end{array}\right]\left[\begin{array}{l}
\theta \\
\dot{\theta} \\
\mathrm{i}
\end{array}\right]+\left[\begin{array}{c}
0 \\
0 \\
\frac{1}{\mathrm{~L}}
\end{array}\right] \mathrm{V} \\
\mathrm{y}=\left[\begin{array}{lll}
1 & 0 & 0
\end{array}\right]\left[\begin{array}{c}
\theta \\
\dot{\theta} \\
\mathrm{i}
\end{array}\right]
\end{gathered}
$$

\section{DRIVE SYSTEM OF MOTORS IN THE SYSTEM:}

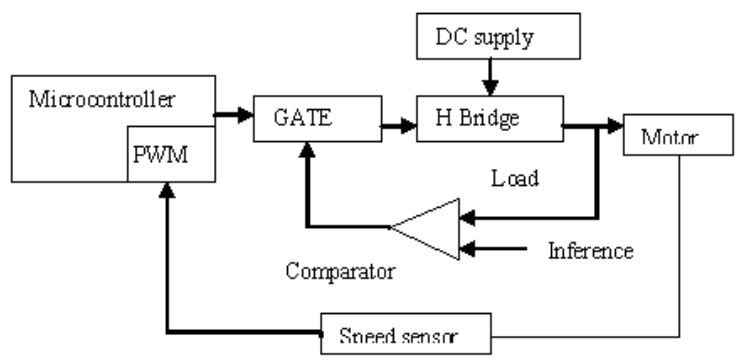

Fig 3: Motor control system

\section{H BRIDGE:}

DC Motors can rotate in two directions depending on how the battery is connected to the motor. In order run the motor in the forward direction, connect the positive terminal of the motor is connected to the positive terminal of the battery and negative to negative. However, to run the motor in reverse just switch the connections; connect the positive terminal of the battery to the negative terminal of the motor and the negative terminal of the battery to the positive terminal of the motor . An H-Bridge circuit allows a large DC motor to be run in both directions with a low level logic input signal. The H-Bridge electronic structure is explicit in the name of the circuit - $\mathrm{H}$ Bridge.

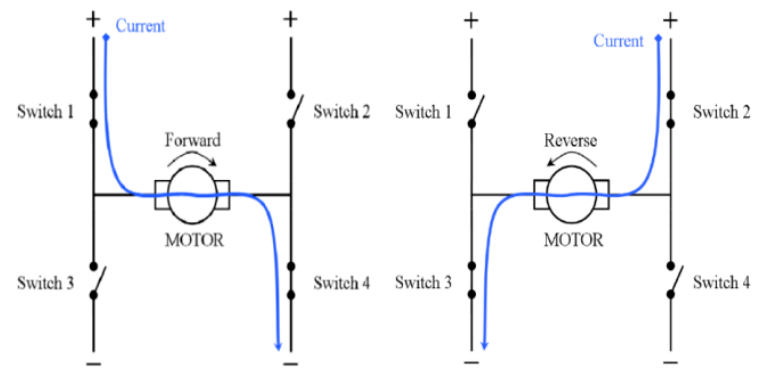

Fig: 4.1

Fig: 4.2

Forward direction $\quad$ Reverse direction

If it is desired to turn the motor on in the forward direction, switches 1 and 4 must be closed to power the motor. Figure 4.1 below is the H-Bridge driving the motor in the forward direction. If it is desired to turn the motor on in the reverse direction, switches 2 and 3 must be closed to power the motor. Figure 4.2 below is the H-Bridge driving the motor in the reverse direction. L293D is a dual $\mathrm{H}$-bridge motor driver, so with one IC we can interface two DC motors which can be controlled in clockwise and anticlockwise direction [2][5].

\section{SPEED CONTROL - PWM TECHNIQUE:}

The motor is controlled by the 4 switches above. For the speed control explanation that follows only switches 1 and 4 will be considered because speed control is identical in the forward and reverse direction. Say the switches 1 and 4 are turned on, the motor will eventually run at full speed. Similarly if only switch 4 is turned on while switch 1 is o. the motor stops. Using this system, how could the motor be run at $1 / 2$ of the full speed? The answer is actually quite simple; turn switch 1 on for half the time and turn it off for the other half. In order to implement this system in reality, one must 
consider two main factors, namely frequency and duty cycle. [7].

\section{FUZZY LOGIC CONTROLLER:}

Prof. L.A. Zadeh developed systematic treatment for Fuzzy Logic controller and later on Mamdani and Assilian used fuzzy sets with an adaptive feedback control strategy to control a small toy steam engine. This was the first practical applications of fuzzy logic controller (FLC). Mamdan applied FLC in the automatic control system of a rotary furnace for cement production after that and later on in the year 1980, Larsen used the fuzzy logic for various industrial applications. For development of FLC in industrial applications first Fuzzy International Conference was held in 1985 in Japan. Yamakawa designed a super high speed fuzzy controller for the Sendai underground railways, which was utilized by Hitachi Company in Japan. This system automatically decreased the speed of a train on entering a station, ensuring that the train stopped at a predetermined place. It also had the benefit of being a highly comfortable ride through mild acceleration and braking. Today, there are number of products in the market which are controlled by fuzzy logic in which different types of FLC are used, the block diagram of the fuzzy logic controller is shown in Figure 5. In general this type of FLC contains four main parts, two of which perform transformations;[4] which are:

a) Fuzzifier (transformation 1)

b) Knowledge base

c) Inference engine

d) Defuzzifier (transformation 2)

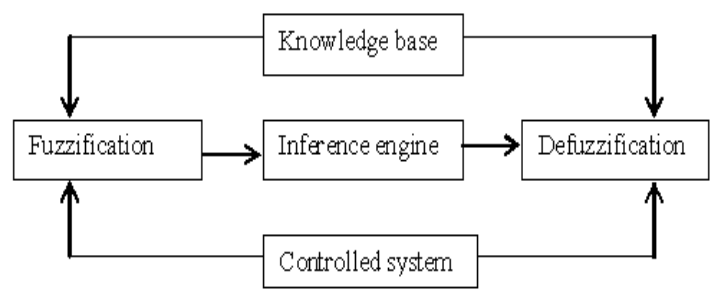

Fig: 5 Fuzzy inference system

Fuzzification measures the values of input variable and converts input data into suitable linguistic values. Knowledge base consist a database and provides necessary definitions, which are used to define linguistic control rules. This rule base characterized the control goals and control policy of the domain experts by means of a set of linguistic control rules. Decision-making logic or inference mechanism is main part of a FLC. It has the capability of simulating human decisionmaking based on fuzzy concepts and of inferring fuzzy control actions employing fuzzy implication and the rules of inference in fuzzy logic. Defuzzification is a scale mapping, which converts the range of values of output variables into corresponding universe of discourse and also yields a nonfuzzy control action from an inferred fuzzy control action. This transformation is performed by Membership Functions (MF). In FLC, number of MF and their shapes are initially determined by user. [8][18].

\section{IMPLEMENTATION OF FLC IN DC MOTOR:}

The controller observes the speed loop error signal and correspondingly updates the controller output so that the actual motor speed matches the reference set speed. There are two input signals to the fuzzy controller, the error $E=$ set speed -actual speed and the derivative of error, CE. In a discrete system, $\mathrm{dE} / \mathrm{dt}=\Delta \mathrm{E} / \Delta \mathrm{t}=\mathrm{CE} / \mathrm{Ts}$, where $\mathrm{CE}=\Delta \mathrm{E}$ in the sampling time Ts. With constant Ts, CE is proportional to $\mathrm{dE} / \mathrm{dt}$. The input variables, error and error rate and output variable, the control action, are represented as linguistic values as follows; $\mathrm{ZE}=$ Zero, $\mathrm{PS}=$ Positive Small, $\mathrm{PM}$ $=$ Positive Medium, $\mathrm{PB}=$ Positive Big NS $=$ Negative Small $\mathrm{NM}=$ Negative Medium, NB =Negative Big [14-15]. After selecting appropriate number of input and output variables and their linguistic values, we have to draw the membership function for these linguistic values. [6].The membership functions of the proposed system are shown below:

\subsection{Membership functions of input and output of fuzzy controller:}

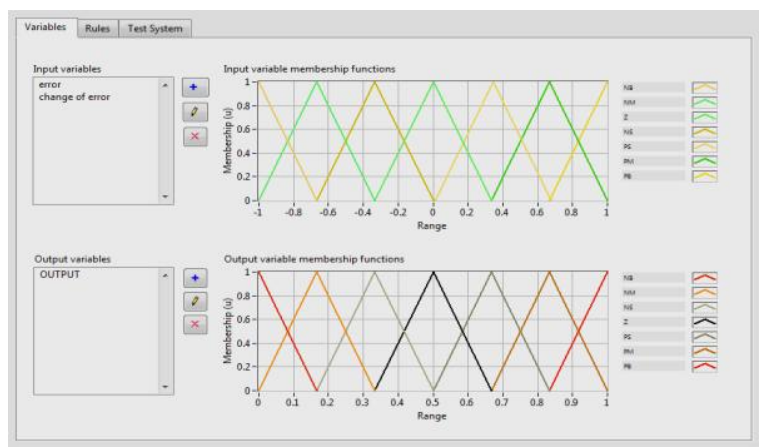

Fig: 6 membership functions of input and output

The triangular membership function for the both input (error, error rate) and output variables are shown in Figure.6. There are seven MFs for inputs e and Ce signals, whereas there are seven MFs for the output. All the MFs are symmetrical for positive and negative values of the variables. Depending on these input variable values, the output variable value is to be decided from the experience encoded in the form of rules. Table 1 shows the corresponding rule table for the speed controller. The top row and left column of the matrix indicate the fuzzy sets of the variables e and $\mathrm{Ce}$, respectively, and the MFs of the output variable (motor torque) operate according to the rule shown in the body of the matrix. [15]. There are $7 \times 7=49$ possible rules in the matrix, where a typical rule reads as:

Table 1.Rule table for speed control

\begin{tabular}{|l|l|l|l|l|l|l|l|}
\hline E & NB & NM & NS & Z & PS & PB & PS \\
\hline NB & PB & PB & PM & PM & PS & PS & Z \\
\hline NM & PB & PM & PM & PS & PS & Z & NS \\
\hline NS & PM & PM & PS & PS & Z & NS & NS \\
\hline Z & PM & PS & PS & Z & NS & NS & NM \\
\hline PS & PS & PS & Z & NS & NS & NM & NM \\
\hline PB & PS & Z & NS & NS & NM & NM & NB \\
\hline PS & Z & NS & NS & NM & NM & NB & NB \\
\hline
\end{tabular}


There are two types of fuzzy inference methods namely Mamdani's method and Sugeno or Takagi-Sugeno-Kang method of fuzzy inference process to calculate fuzzy output [7][8]. The Mamdani implication is found suitable for DC machine and induction machine models. In order to convert fuzzy output in to a crisp value of the output variable, the defuzzification process is employed. The centre of area (COA) de-fuzzification method is generally used. Using this method, the centroid of each output membership function for each rule is first evaluated. The final output torque is then calculated as the average of the individual centroids, weighted by their heights (degree of membership). The fuzzy logic controller output torque is applied to the PWM. The PWM controls the desired speed of the motor can be obtained. Data Socket is a technology that simplifies data exchange between an application and other applications, files, FTP servers, and Web servers. It provides one common API to a number of different communication protocols.[14].

\section{DIRECTION CONTROL OF THE ROBOT:}

The robot can be moved in forward direction by running both the motors in clockwise direction in set speed and the robot can be moved in backward direction by running both the motors in anticlockwise direction. The robot is moved in leftwards or rightwards when the obstacle is detected, by running one motor in clockwise direction with high set speed and the other in anticlockwise direction with low set speed (almost zero rpm) and one motor in anticlockwise direction with high set speed and other in clockwise direction with low set speed(almost zero rpm) respectively.

Table 2. Robot direction

\begin{tabular}{|l|l|l|}
\hline Direction & Left Motor & Right Motor \\
\hline Forward & Clockwise & Clockwise \\
\hline Backward & Anticlockwise & Anticlockwise \\
\hline Right & Clockwise & Stop \\
\hline Left & Stop & clockwise \\
\hline Stop & Stop & Stop \\
\hline
\end{tabular}

\section{DATA SOCKET TRANSFER PROTOCOL:}

Data Socket greatly simplifies this task by providing a unified API for these low-level communication protocols. Transferring data across computers with Data Socket is as simple as using a browser to read Web pages on the Internet.

Data Socket Transfer Protocol based on TCP/IP through which data is passed between Data Socket clients_known as Data Socket Reader and Writer applications- using the Data Socket Server.Once connected to a Data Socket Server from Data Socket Reader and Writer applications using the DSTP URL scheme, as demonstrated in the following example URLs.The following URL connects to the data item named wave on the Data Socket Server running on the local computer:dstp://localhost/wave The following URL connects to the data item named wave on a Data Socket Server running on a networked computer named lab:dstp://lab/wave.

\subsection{Server Data Socket Program Implementation:}

The Data Socket protocol based program in the server computer opens bi-directional communication using Data Socket Open Connection sub VI. The URL of the server is selected as dstp://localhost/file_name. The server receives set point information from the client as a double precision value. 1 . The client controls the set-speed on the server.

\subsection{Client Data Socket Program Implementation:}

The client computer also opens bi-directional communication using Data Socket Open Connection sub VI. The URL of the server is selected as dstp://ip_address of server/file_name. This URL allows connection between clients and the server using Data Socket protocol to process the information. The client sends set-speed information to the server.

\section{GPS:}

GPS is used for positioning, locating and determining time. The civil signal SPS (Standard Positioning Service) can be used freely by the general public, whilst the Military signal PPS (Precise Positioning Service) can be used only by authorized government agencies. The GPS data can be retrieved from GPS receiver in various formats. GPRMC data format is used in the proposed system. The RMC data Set (Recommended Minimum Specific GNSS) contains information on time, latitude, longitude and height, system status, speed, course and date.

\section{Table 2.GPRMC Format}

\begin{tabular}{|l|l|}
\hline Field & Description \\
\hline$\$$ & Start of the data set \\
\hline GP & Information originating from a GPS appliance \\
\hline RMC & Data set identifier \\
\hline 130304.0 & Time of reception (world time UTC): $13 \mathrm{~h} 03 \mathrm{~min} 04.0 \mathrm{sec}$ \\
\hline A & Data set quality: A signifies valid (V= invalid) \\
\hline 4717.115 & Latitude: $47^{\circ} 17.115$ min \\
\hline $\mathrm{N}$ & Northerly latitude ( $\mathrm{N}=$ north, $\mathrm{S}=$ south) \\
\hline 00833.912 & Longitude: $8^{\circ} 33.912$ min \\
\hline E & Easterly longitude ( $\mathrm{E}=$ east, $\mathrm{W}=$ west) \\
\hline 000.04 & Speed: 0.04 knots \\
\hline 205.5 & Course: $205.5^{\circ}$ \\
\hline 200601 & Date: 20 th June 2001 \\
\hline 01.3 & Adjusted declination: $1.3^{\circ}$ \\
\hline W & Westerly direction of declination $(\mathrm{E}=$ east) \\
\hline$\star$ & Separator for the checksum \\
\hline $7 C$ & Checksum for verifying the entire data set \\
\hline$<$ CR $>$ <LF $>$ & End of the data set \\
\hline
\end{tabular}

\section{SYSTEM IMPLEMENTATION:}

The proposed system can be implemented with the help of pic controller 16f877A with $20 \mathrm{MHz}$ clock input and 9v Dc motor consuming 2.7 Amps having $4100 \mathrm{rpm}$. H Bridge is implemented by L293d driver IC, to drive the motors.GPS is attached to the external USART of the controller. Regulator 7805 and 7809 is used to regulate the voltage to controller, sensors and motors.

\section{RESULTS AND CONCLUSION:}

The results of the implemented system are shown from figure 12.1 to 12.8 .

For the assembly software programming of the control circuit, the process is very simply and the procedure can be mentioned as the following steps. 
Initialization

Ports Declaration: all Port A's pins are declared as inputs,

four MSB Port B pins are declared as outputs.

Start program:

Check RA4 (MSB) high or low

Check RA3 high or low

Check RA2 high or low

Check RA1 high or low

Check RA0 high or low

Determined outputs:

- If all inputs data are low outputs RB0-RB3, "1010" for freely going forward.

- If metal sensor RA0 and RA1 are high, outputs "1111" to stop. Gps data is obtained from external UART.

\subsection{Pwm signal generation:}

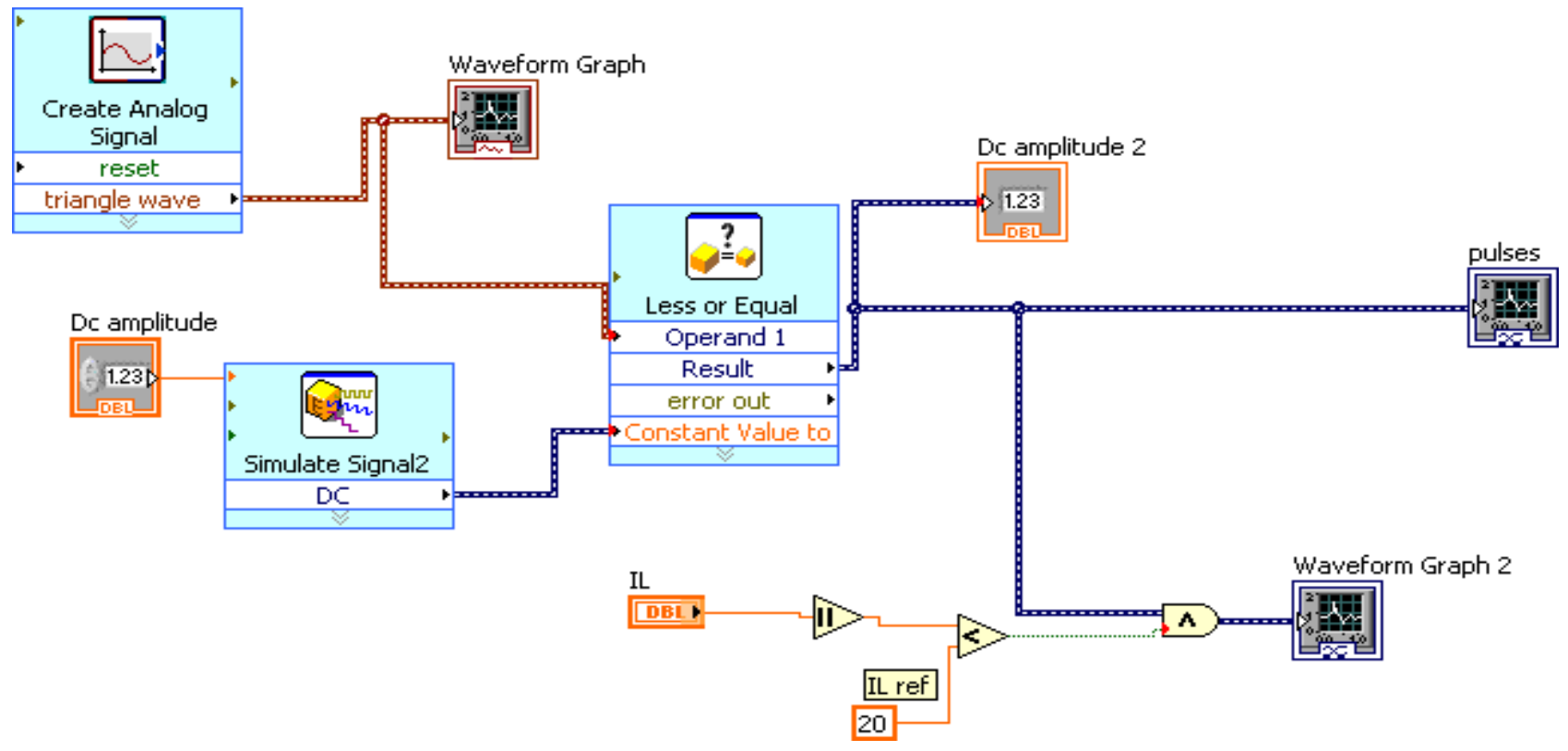

Fig: 13.1 Block diagram of pwm signal generation

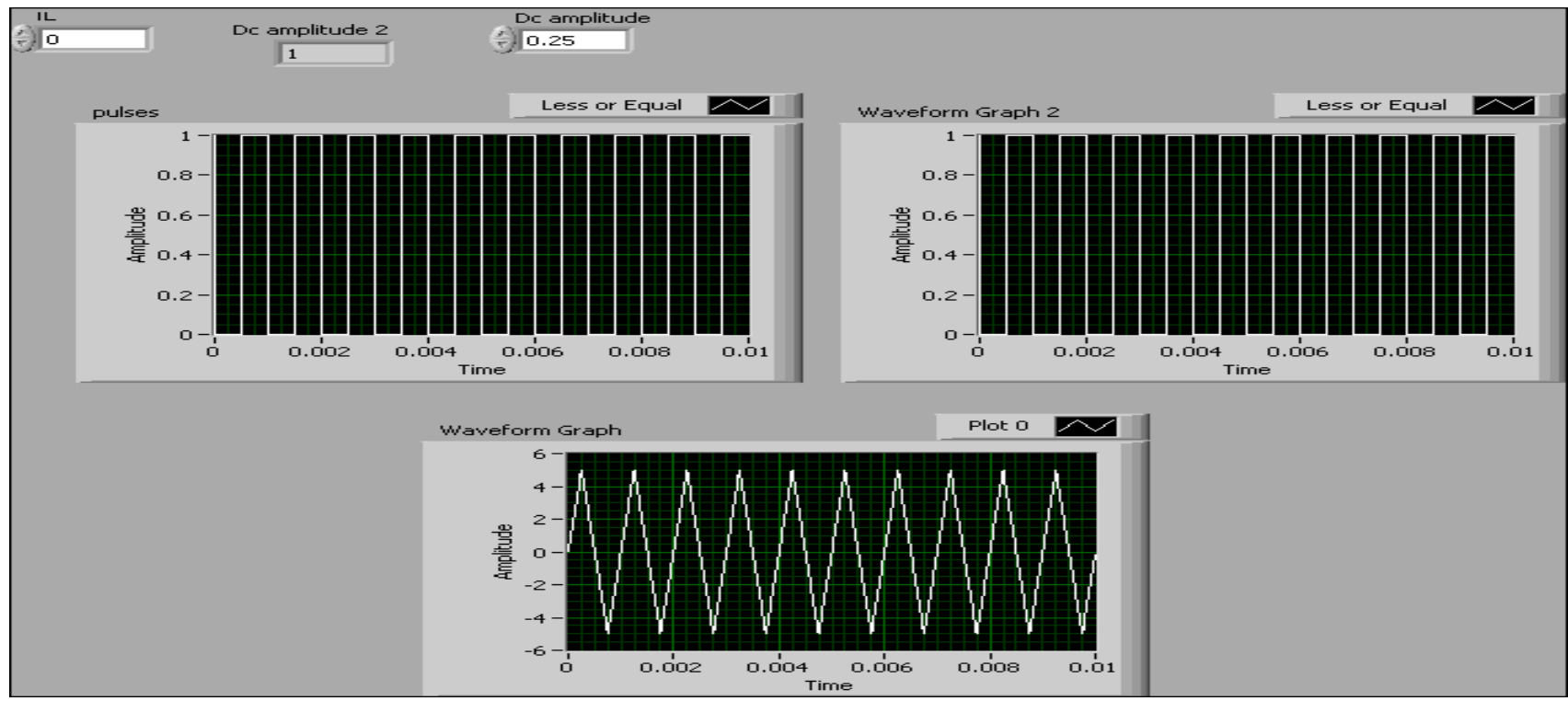

13.1 Front panel of pwm signal generation 
The above graph Fig. 12.1 shows the PWM signal generated to control the speed of the motor. This required PWM signal is generated from fuzzy controller. This signal is fed to $\mathrm{h}$ bridge to on and off the switches according to the directions.

\subsection{Fuzzy controller:}

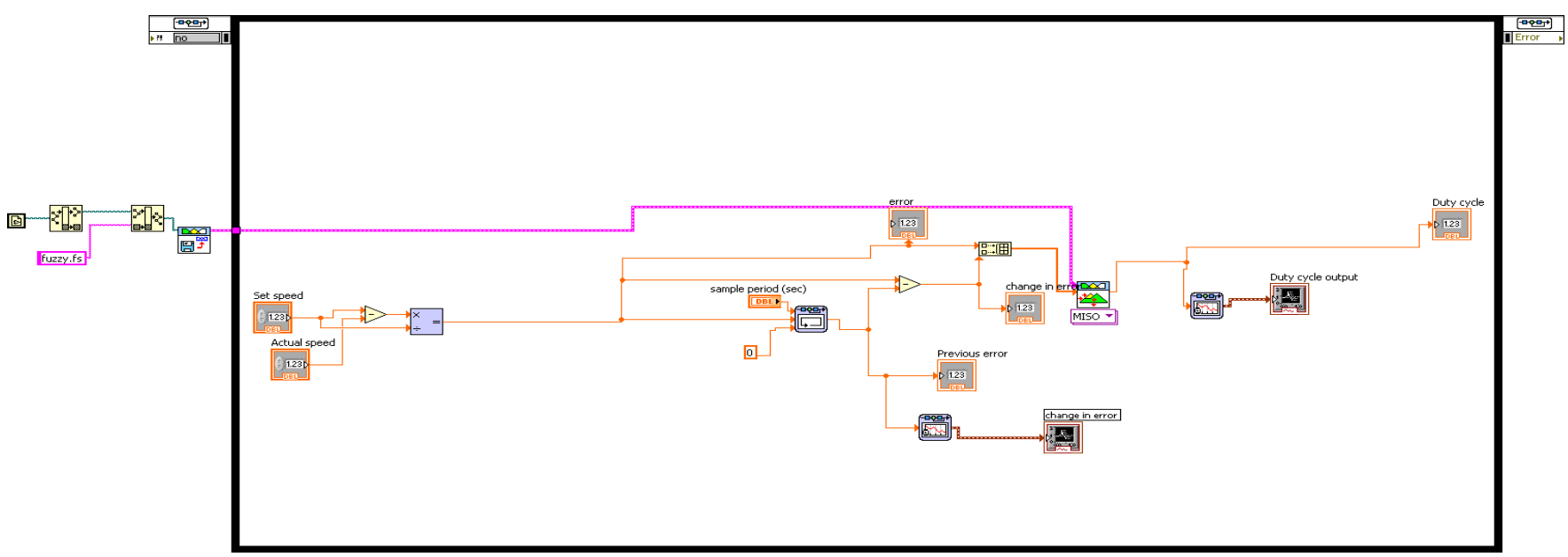

Fig: 13.2 Block diagram of fuzzy controller

\subsection{DCmotor:}

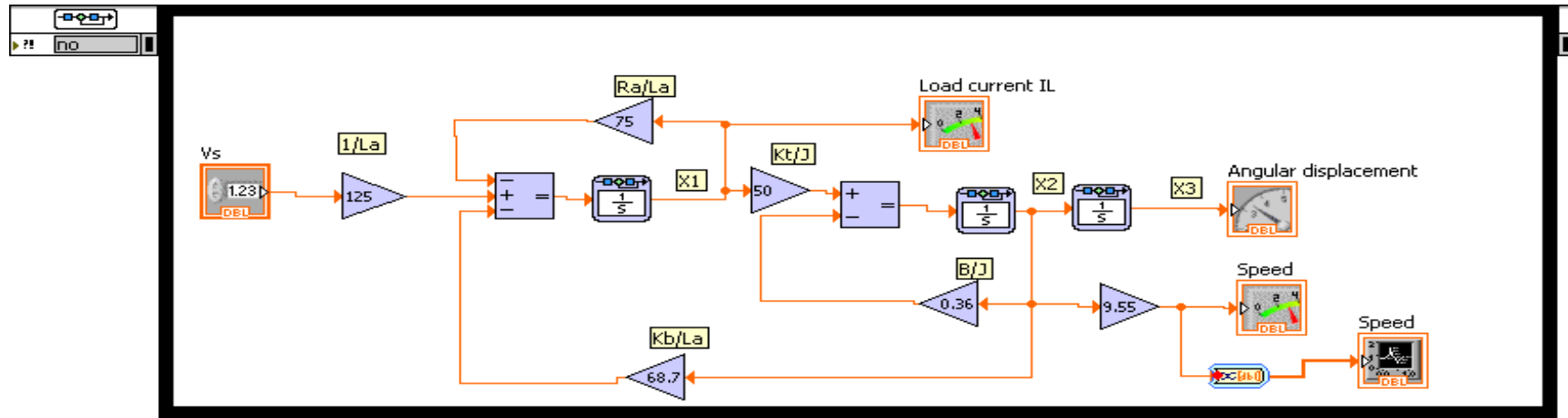

Fig: 13.3 Block diagram of DC motor

\subsection{Speed response of dc motor using fuzzy controller:}

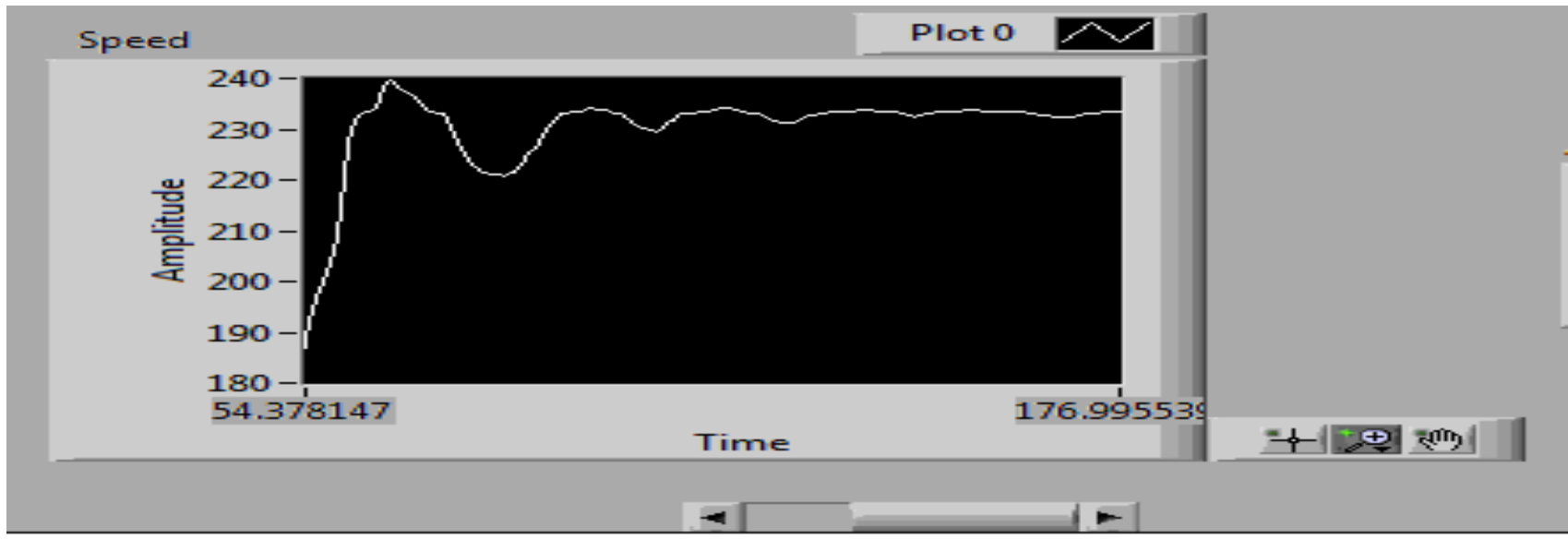

Fig :13.4 Front panel of speed response of DC motor using fuzzy controller for set speed of $235 \mathrm{rpm}$

The above graph Fig. 12.4 shows the speed response of the motor. The motor runs at $100 \mathrm{rpm}$. The set speed is obtained via h bridge and fuzzy controller. Similarly the motion of the robot can be controlled various set speeds. 


\subsection{Online control of the system using data socket protocol:}

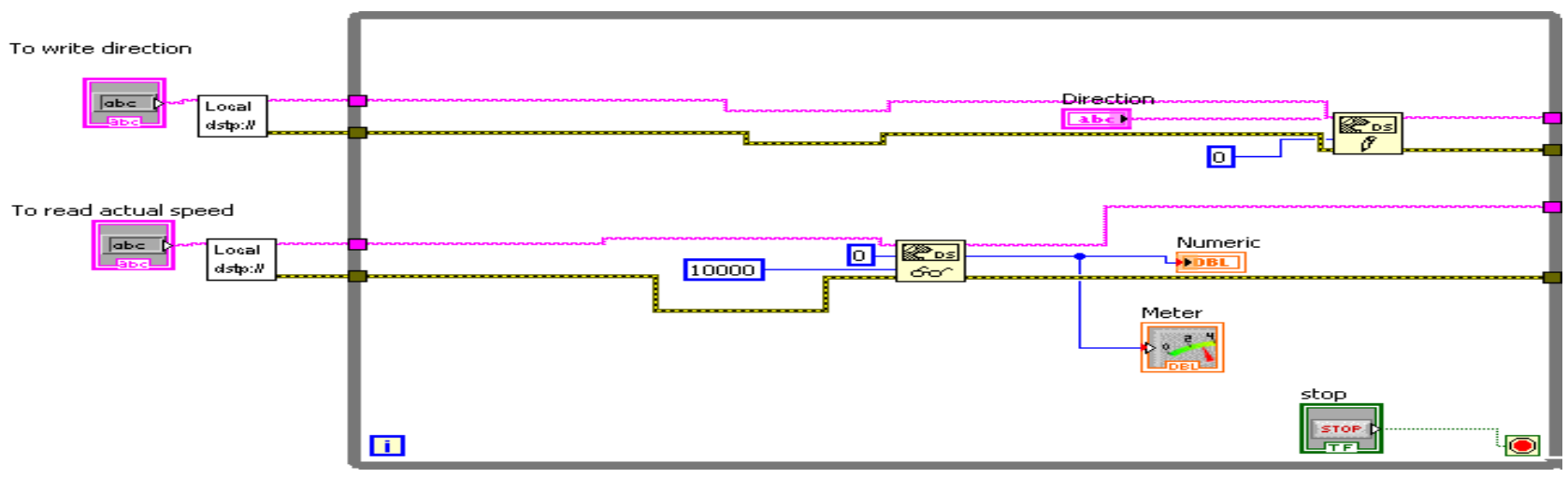

Fig: 13.5 Block diagram of online data transfer using data socket protocol

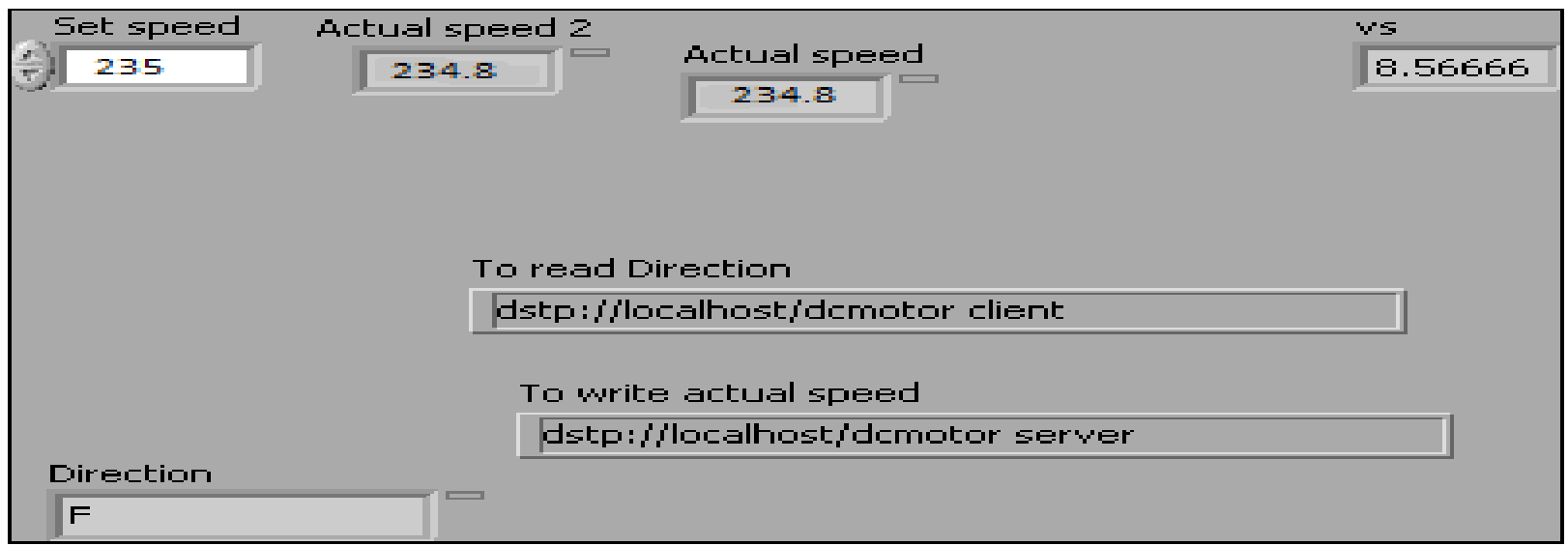

Fig: 13.5 Front panel of online data transfer using data socket protocol

Fig. 13.5 shows the online control of robot via data socket protocol. The set speed is written to the microcontroller (robot) and the direction of the motor is read from robot to the server system.

This paper presents the complete application of mine detection. The advantage of the implemented system is video monitoring of the robot. This reduces damage to the robot .This paper can be further extended by using obstacle sensor on all four sides and decides direction automatically.

\section{REFERENCES}

[1] Gopal, M., "Modern Control System Theory", 2nd ed., Wiley Eastern Ltd., 1993.

[2] Krishnan, R. "Electric Motor Drives, Modeling, Analysis and Control", 1st ed., Singapore: Pearson Education, 2001.

[3] P. C Sen, "Principles of Electric Machines and Power Electronics", 3rd ed., New York: Wiley, 1988.

[4] B. Kosko, "Neural Networks and Fuzzy Systems: A Dynamical Systems Approach to Machine Intelligence", Prentice-Hall Inc., 1992.

[5] W. Leonhard, "Control of Electric Drives", 3rd ed., New York: Springer, 2001.

[6] S. N. Sivanandam, S. Sumathi, and S. N. Deepa, "Introduction to Fuzzy Logic using MATLAB", Berlin
Heidelberg: Springer-Verlag, ISBN-13 978-3-540-57803, 2007.

[7] Bose, B. K. "Expert system, fuzzy logic, and neural network applications in power electronics and motion control," Proc. IEEE, Vol. 82, No. 8, Aug.1994, pp. 1303-1323.

[8] Lee, J. "On methods for improving performance of PI-type fuzzy logic controllers," IEEE Trans. on Fuzzy Systems, vol. 1, No. 4Nov. 1993. , pp. 298302

[9] P. Yedamale, Brushless DC (BLDC) Motor Fundamentals. Chandler, AZ:Microchip Technology, Inc., last access; March 15,2009.

[10] B.K. Lee and M. Ehsani, Advanced Simulation Model for Brushless DC Motor Drives, Electric Power Components and Systems 31, 841-868, 2003.

[11] W. Hong, W. Lee, and B.K. Lee, Dynamic Simulation of Brushless DC Motor Drives Considering Phase Commutation for Automotive Applications, ElectricMachines \& Drives Conference, IEMDC '07, 2007. 
[12] R. Carlson, M. Lajoie-Mazenc, and C.D.S. Fagundes, Analysis of torque ripple due to phase commutation in brushless DC machines, IEEE Trans. on Industry Applications 28, 632-638, 1992.

[13] S.K. Safi, P.P. Acarnley, and A.G. Jack, Analysis and simulation of the high-speed torque performance of brushless DC motor drives, Proc. of the IEE 142, 191200, 1995.

[14] CK. Lee and WH. Pang, A Brushless DC Motor Speed Control System Using Fuzzy Rules, IEE Power Electronics and Variable Speed Drives, pp.101-106, 1994.

[15] R.N. Tuncay, Z. Erenay, M. Yýlmaz, and Ö. Üstün, Rapid Control Prototyping Approach to Fuzzy Speed
Control of Brushless DC Motor, ELECO'03,International Conference on Electrical and Electronics Engineering, Bursa,Turkey, 2003.

[16] P.D. Evans and D. Brown, Simulation of brushless DC drives, Proc. of the IEEE 137, 299-308, 1990.

[17] C.W. Hung; C.T. Lin, and C.W. Liu, An Efficient Simulation Technique for the Variable Sampling Effect of BLDC Motor Applications, IECON 2007, pp. 11751179, 2007.

[18] C. Xia, P. Guo, T. Shi, and M. Wang, Speed control of brushless dc motor using genetic algorithm based fuzzy controller, Proc. of Int. Conf. on IntelligentMechatronics and Automation, pp.460-464, 2006. 